\title{
Estudos taxonômicos em Gloxinieae (Gesneriaceae) - notas nomenclaturais
}

Andréa Onofre de Araujo ${ }^{1,4}$, Vinicius Castro Souza ${ }^{2}$ e Alain Chautems ${ }^{3}$

Recebido em 4/04/2012. Aceito em 18/07/2012

\section{RESUMO}

(Estudos taxonômicos em Gloxinieae (Gesneriaceae) - notas nomenclaturais). Gloxinia L'Hér. foi um dos gêneros cuja delimitação foi mais modificada em decorrência da reorganização da tribo Gloxinieae nas últimas décadas. Como parte dos estudos taxonômicos no gênero, são designados 15 lectótipos, indicados seis nomes ilegítimos supérfluos, e proposta uma nova combinação neste trabalho; são incluídos, também, comentários sobre três espécies com identidade incerta.

Palavras-chave: nomenclatura, Gloxinella, Goyazia, Mandirola, Seemannia

\begin{abstract}
(Taxonomic studies on Gloxinieae (Gesneriaceae) - nomenclatural notes). Gloxinia L'Her. is a genus that has undergone major changes since the reorganization of the tribe Gloxinieae in the last few decades. We here designate 15 lectotypes, indicate six illegitimate superfluous names, and propose one new combination for this group; comments about three species with uncertain identities are also included.
\end{abstract}

Key words: nomenclature, Gloxinella, Goyazia, Mandirola, Seemannia

\section{Introdução}

Análises filogenéticas (Zimmer et al. 2002; Roalson et al. 2005a) mostraram que vários gêneros da tribo Gloxinieae (Gesneriaceae) não eram monofiléticos, estimulando modificações profundas na taxonomia do grupo. Nas últimas décadas, alguns de seus gêneros tiveram sua circunscrição modificada, outros foram restabelecidos ou excluídos da tribo e gêneros novos foram descritos (Roalson et al. 2005b; Araujo et al. 2010a). Com essas reformulações, Gloxinieae passou a contar com 20 gêneros; Gloxinia L'Hér. foi um gênero drasticamente reformulado, passando de 15 espécies (Wiehler 1976, 1983) para apenas quatro: G. perennis (L.) Fritsch, G. erinoides (DC.) Roalson \& Boggan, G. xanthophylla (Poeppig) Roalson \& Boggan - as duas últimas eram antes incluídas nos gêneros monotípicos Koellikeria Regel e Anodiscus Benth., respectivamente - e G. alterniflora A.O.Araujo \& Chautems, descrita recentemente (Araujo et al. 2010b). Com exceção de G. perennis (espécie-tipo), as demais espécies foram acomodadas por Roalson et al. (2005b) em sete gêneros: Gloxinella (H.E.Moore) Roalson \& Boggan [G. lindeniana (Regel) Roalson \& Boggan], Gloxiniopsis Roalson \& Boggan [G. racemosa (Benth.) Roalson \& Boggan], Mandirola Decne. [Mandirola ichthyostoma (Gardner) Seem. ex Hanst., M. multiflora (Gardner) Decne. e M. rupestris (Gardner) Roalson \& Boggan], Monopyle Moritz ex Benth. [M. reflexa (Rusby) Roalson \& Boggan], Nomopyle Roalson \& Boggan [N. dodsonii (Wiehler) Roalson \& Boggan], Seemannia Regel [S. gymnostoma (Griseb.) Toursark., S. nematanthodes (Kuntze) K.Schum., S. purpurascens Rusby e S. sylvatica (Kunth) Hanst.] e Sphaerorrhiza Roalson \& Boggan [S. burchellii (S.M.Phillips) Roalson \& Boggan e $S$. sarmentiana (Gardner ex Hook.) Roalson \& Boggan], o qual foi incluído em uma nova tribo monogenerica (Sphaerorrhizeae); além disso, Gloxinia villosa (Gardner) Wiehler foi tratada como incertae sedis.

\footnotetext{
${ }^{1}$ Universidade Federal do ABC, Centro de Ciências Naturais e Humanas, Santo André, SP, Brasil

${ }^{2}$ Universidade de São Paulo, Escola Superior de Agricultura "Luiz de Queiroz", Departamento de Ciências Biológicas, Piracicaba, SP, Brasil

${ }^{3}$ Conservatoire \& Jardin Botaniques de la Ville de Genève, Genebra, Suíça

${ }^{4}$ Autor para correspondência: andrea.onofre@ufabc.edu.br
} 
Durante a revisão taxonômica de Gloxinieae, incluindo a maior parte das espécies consideradas por Wiehler (1976, 1983) em Gloxinia, Araujo (2007) ampliou a compreensão sobre os relacionamentos internos na tribo, reconheceu novos táxons e aprimorou a circunscrição das espécies. Como resultado desses progressos, dados filogenéticos e taxonômicos já foram publicados (Araujo et al. 2010a,b) e outros estão em preparação. Buscando adequar a nomenclatura de Gloxinieae às recentes novidades na sistemática da subfamília, são designados 15 lectótipos, indicados seis nomes ilegítimos supérfluos e proposta uma nova combinação neste trabalho; são incluídos, também, comentários sobre três espécies com identidade ainda incerta.

\section{Material e métodos}

Foram consultadas as obras originais das espécies e coleções de 40 herbários: B, BHCB, BM, BR, CEN, CGE, E, ESA, ESAL, F, G, GZU, HB, HBG, HPL, HTO, HUEFS, IBGE, IAN, IPA, K, L, LE, MBM, MG, MO, NY, P, R, RB, S, SP, SPF, U, UB, UFG, US, W, WAG, Z. Representantes dessas espécies foram observados no campo e/ou em cultivo: Sítio Primavera, em Mogi das Cruzes, e Instituto Plantarum, em Nova Odessa, ambas as coleções no estado de São Paulo; e Jardim Botânico de Genebra, Suíça.

\section{Tratamento taxonômico}

1. Gloxinella (H.E.Moore) Roalson \& Boggan, Selbyana 25(2): 227. 2005. Kohleria sect. Gloxinella H.E.Moore, Gentes Herb. 8: 382. 1954. Tipo: G. lindeniana (Regel) Roalson \& Boggan (Tydaea lindeniana Regel).

1.1. Gloxinella lindeniana (Regel) Roalson \& Boggan, Selbyana 25(2): 227. 2005. Tydaea lindeniana Regel, Gartenflora 17: 257; pl. 589. 1868. Isoloma lindenianum (Regel) G.Nicholson, Ill. Dict. Gard. 2: 201. 1885. Gloxinia lindeniana (Regel) Fritsch, Oesterr. Bot. Z. 63: 66. 1913. Kohleria lindeniana (Regel) H.E.Moore, Gentes Herb. 8: 380. 1954. Tipo: Equador, ex horto Petropolitano, 68.3 (Holótipo LE). Tydaea lindenii Regel ex André, Ill. Hort. 20: 183; pl. 147.

1873, nom. illeg. superfl.

Distribuição: Equador (?) e Peru.

Essa espécie havia sido coletada uma única vez na natureza, sem localidade precisa, e foi levada para cultivo na Europa há mais de 100 anos. Sua descrição original está provavelmente baseada nesta planta em cultivo e em um material de herbário pobremente representado. Atualmente, ela é amplamente cultivada no Brasil (e.g., Instituto Plantarum, Nova Odessa - SP, e no Sítio Primavera, Mogi das Cruzes - SP) e no exterior (e.g., Jardim Botânico de Genebra, Suíça, e Marie Selby Botanical Gardens, Flórida, EUA). Sua ocorrência no Equador não foi confirmada, porém uma ocorrência no Peru foi incluída no "World Checklist of
Gesneriaceae" (Skog \& Boggan, 2011). Essa informação tem como base duas coletas da espécie em Cajamarca, realizadas nos anos de 1996 e 1997 (Campos \& Diaz 2467 e Campos et al. 3341), ambas depositadas nos herbários US e MO e identificadas pelo Dr. L.E. Skog (John Boggan, com. pess.).

O material-tipo de Tydaea lindeniana estaria depositado no herbário LE, já que o autor trabalhava no Jardim Botânico Imperial de São Petersburgo quando descreveu a espécie. $\mathrm{Na}$ obra original de T. lindeniana, é mencionado que o material foi levado da América tropical por Linden, que a ilustração foi preparada pelo próprio coletor e que o autor analisou uma flor seca e uma folha, além de ter observado a planta que estava em cultivo com Linden. Regel geralmente preparava exsicatas de material em cultivo, mencionando "Ex horto bot. Petropolitano" e com uma numeração que parece conter a década (nos anos 1800), seguindo um número sequencial, geralmente entre 1 e 100. Em visita ao herbário LE, verificamos que existe uma exsicata com uma flor seca e uma folha, consultada por Regel e com o número "68.3", correspondendo ao holótipo de T. lindeniana; o número "68.3" provavelmente indica o ano de 1868. Regel (1868) comentou que Hanstein havia denominado essa espécie de Gloxinia tydaeoides (um nomen nudum, apenas citado nessa obra de Regel) e enviou um exemplar seco para Linden. Regel não concordou com o posicionamento de Hanstein, pois a planta apresentaria rizoma permanente escamoso e um tipo de indumento que o levou a incluí-la em Tydaea. O nome Tydaea lindenii trata-se de um nome supérfluo para Tydaea lindeniana, pois André (1873), ao descrevê-la, comentou que a espécie havia sido nomeada "Tydaea lindeni" por Regel, conforme informado por J. Linden. Além disso, ele (André 1873) comentou que a descrição e a ilustração em sua obra estavam baseadas no material vivo do horto de Linden, mesma origem do material utilizado por Regel na descrição dessa espécie, e que a origem da planta cultivada seria o Equador. No entanto, ele não sabia como, nem onde a espécie havia sido publicada por Regel, referindo-se a ela como Tydaea lindenii.

Pode parecer surpreendente que até agora essa espécie permaneça sem registros no Equador, apesar das numerosas expedições de coleta naquele país por vários especialistas de Gesneriaceae, como H. Wiehler e J.L. Clark, a partir dos anos de 1980.

Material selecionado: Brasil. São Paulo: Instituto Plantarum (cultivada, sem procedência), Nova Odessa, 19/ III/2004, A.O. Araujo 463 (ESA).

2. Gloxinia L'Hér. in Aiton, Hort. Kew. 2: 331. 1789. Tipo: G. maculata L'Hér., nom. illeg. superfl. [= G. perennis (L.) Fritsch]

Eucolum Salisb., Prodr. Stirp. Chap. Allerton: 98. 1796. Tipo: E. crassifolium Salisb., nom. illeg. superfl. [= G. perennis (L.) Fritsch]

Koellikeria Regel, Index Sem. (Zurich): 4. 1847. Tipo: K. argyrostigma (Hook.) Regel [= Gloxinia erinoides (DC.) Roalson \& Boggan]. 
Escheria Regel, Flora 32: 179. 1849, nom. inval. Tipo: E. gloxiniiflora Regel, nom. illeg. superfl. [= G. perennis (L.) Fritsch]

Salisia Regel, Flora 32: 179. 1849 (non Salisia Lindl. 1839 = Myrtaceae). Tipo: S. gloxiniiflora Regel, nom. illeg. superfl. [= Gloxinia perennis (L.) Fritsch]

Anodiscus Benth., Gen. Pl. 2: 998. 1876. Tipo: A. peruvianus Benth. [= Gloxinia xanthophylla (Poepp.) Roalson \& Boggan]

Além de um homônimo posterior, Salisia Regel também é um nome supérfluo, pois Regel (1849) citou Gloxinia maculata, a espécie-tipo de Gloxinia, dentre as espécies do gênero, semelhante ao que aconteceu na descrição de Eucolum.

2.1. Gloxinia erinoides (DC.) Roalson \& Boggan, Selbyana 25(2): 228. 2005. Achimenes erinoides DC., Prodr. 7: 536. 1839. Trevirana erinoides (DC.) D.Dietr., Syn. Pl. 3: 568. 1843. Diastema erinoides (DC.) Benth. ex Walp., Repert. Bot. Syst. 6: 403. 1847. Koellikeria erinoides (DC.) Mansf., Repert. Spec. Nov. Regni Veg. 38: 28. 1935. Tipo: Venezuela, Districto Federal, s.d., J.M. Vargas 1630 (Holótipo G-DC! [G00133345]).

Achimenes argyrostigma Hook., Bot. Mag. 71: pl. 4175. 1845. Koellikeria argyrostigma (Hook.) Regel, Index Sem. (Zurich) 1847: 4. 1847. Tipo: Colômbia, Sierra Nevada de Santa Marta, New Grenada, VII/1844, W. Purdie s.n. (Holótipo K!; Isótipos CGE!, E!).

Koellikeria major Fritsch, Bot. Jahrb. Syst. 50: 398. 1913. Tipo: Bolívia, Chuquisaca, 31/I/1904, K. Fiebrig 2680 (Lectótipo, aqui designados: G!; Isolectótipos F!, GH).

Distribuição: Cerrados e campos rupestres do Planalto Central Brasileiro, sendo raramente encontrada em cerrados das Regiões Norte e Nordeste. Encontrada, também, na Venezuela, Nicarágua e próximo à costa leste dos Andes, na Bolívia, Peru e Colômbia.

Como o holótipo de Koellikeria major, depositado no herbário B, foi destruído durante a guerra, o material Fiebrig 2680, depositado no herbário G, está sendo designado como lectótipo por estar completo, bem preservado e refletir perfeitamente a descrição original da espécie.

Material selecionado: BRASIL. Goiás: Caiapônia, estrada de terra Caiapônia-Montividiu, fazenda Kalosmi, 1704'876S, 51³7’736W, 740 m s.n.m., 16/I/2005, A.O. Araujo et al. 501 (ESA).

2.2. Gloxinia perennis (L.) Fritsch, Nat. Pflanzenfam. 4(3b): 174. 1894. Martynia perennis L., Sp. Pl. (ed. 1) 2: 618. 1753. Tipo: Hort. Cliff. 322: pl. 18. 1737! (Lectótipo, designado por Stearn 1957).

Gloxinia maculata L'Hér. in Aiton, Hort. Kew. 2: 331. 1789, nom. illeg. superfl.

Eucolum crassifolium Salisb., Prodr. Stirp. Chap. Allerton: 98. 1796, nom. illeg. superfl.
Gloxinia trichotoma Moench, Suppl. Meth.: 194. 1802, nom. illeg. superfl.

Gloxinia heterophylla Poepp., Nov. Gen. Sp. Pl. 3: 9. 1840. Tipo: Peru, Peruvia subandina, crescit in sylvis Peruviae lucidioribus, versus Pampayaco, praeruta argillosa colens, I/1829, E.F. Poeppig 1619 (Holótipo W!).

Gloxinia pallidiflora Hook., Bot. Mag. 72: pl. 4213. 1846. Tipo: Bot. Mag. 72: pl. 4213. 1846 (Lectótipo, aqui designado).

Escheria gloxinifflora Regel, Flora 32: 179. 1849 (como 'gloxiniaeflora'), pro syn.

Gloxinia bicolor Poepp. ex Hanst. in C.F.P. Martius, Fl. Bras. 8(1): 348. 1864, pro syn.

Gloxinia trichantha Miq., Linnaea 22: 473. 1849. Tipo: Suriname, in hortis surinamensibus culta et in arenosis spontanea, s.d., H. Focke 822 (Holótipo U!).

Salisia gloxiniiflora Regel, Flora 32: 179. 1849 (como 'gloxiniaeflora'), nom. illeg. superfl.

Distribuição: É a espécie do gênero com distribuição mais ampla, ocorrendo desde a Floresta Atlântica do Sudeste do Brasil, porém sem confirmação de que se tratam de populações nativas dessa formação vegetal, alcançando florestas de galeria em cerrados do Planalto Brasileiro, com poucas ocorrências na Amazônia, avançando pela face leste dos Andes até alcançar a região do Caribe, desde a Venezuela até a Guiana Francesa; alcança também a América Central, do Panamá a Honduras.

Wiehler (1976) incluiu Gloxinia heterophylla, G. pallidiflora, G. suaveolens Decne. e G. trichantha na sinonímia de G. perennis; a sinonimização de $G$. suaveolens não foi aceita no presente trabalho e a identidade desta espécie ainda é incerta (ver comentários a seguir). Outros sinônimos referidos por ele correspondem a nomes ilegítimos e um nomen nudum.

Existe um espécime de $M$. perennis no herbário BM citado como material-testemunho (Herb. Clifford: 322), contando com duas flores e uma folha. No entanto, este material não foi citado na obra original, o que levou Stearn (1957) a designar a prancha citada no protólogo como lectótipo deste nome.

Gloxinia maculata é um nome supérfluo, pois quando L'Héritier (1789) descreveu o gênero Gloxinia citou Martynia perennis como sinônimo. Gloxinia bicolor foi citado por Hanstein (1864) como sinônimo de Gloxinia maculata: "Gloxinia bicolor Poepp. Mss. in Herb.", indicando que este nome deve ter sido usado por Poeppig em etiqueta de herbário, tratando-se de um nomen nudum e publicado em sinonímia. Eucolum crassifolium e Gloxinia trichotoma foram outros dois casos de nomes supérfluos, pois Gloxinia maculata e Martynia perennis foram consideradas na sinonímia no protólogo dessas espécies.

Regel (1849) publicou Salisia gloxiniiflora (originalmente, "gloxiniaeflora") sem citar nenhum material e incluiu Gloxinia maculata, G. trichotoma, Martynia perennis e Escheria gloxi- 
niaeflora como sinônimos. Dessa maneira, Salisia gloxiniiflora trata-se de um nome ilegítimo supérfluo. Um material depositado no herbário Z possui a anotação "Escheria gloxiniaeflora (mihi)" com a letra de Regel, coletado por ele e com o número 48-9, provavelmente setembro de 1848. Entretanto, Escheria gloxiniiflora foi publicada em sinonímia, tratando-se de um nome inválido, não carecendo de lectotipificação.

Na descrição original de Gloxinia pallidiflora, Hooker (1846) mencionou que o material havia sido enviado de Santa Marta, Colômbia, por W. Purdie (coletor de Kew), e que a planta havia florescido em cultivo, em outubro de 1845. Entretanto, ele não deve ter feito exsicatas dessa planta, mantendo-a apenas em cultivo, pois nenhum material que correspondesse ao espécime descrito por Hooker foi localizado. Dessa forma, a prancha 4213, presente no protólogo, está sendo aqui designada como lectótipo de G. pallidiflora.

Material selecionado: BRASIL. Pará: Conceição do Araguaia, ca. $10 \mathrm{~km}$ a oeste de Conceição, em direção a Redenção, Serra no sentido N-S, em estrada de terra à direita da BR, 08¹5»25»S, 49²2»19,8»W, 265 m s.n.m., 29/I/2005, A.O. Araujo 536 (ESA).

2.3. Gloxinia xanthophylla (Poepp.) Roalson \& Boggan, Selbyana 25(2): 228. 2005. Gesneria xanthophylla Poepp in Poeppig \& Endlicher., Nov. Gen. Sp. Pl. 3: 7. 1840. Anodiscus xanthophyllus (Poepp.) Mansf., Repert. Spec. Nov. Regni Veg. 36: 124. 1934. Tipo: Peru, Peruvia subandina, in montibus aridis Chihuamecla, Cuchero, 1829, E.F. Poeppig s.n. (Holótipo W!).

Anodiscus peruvianus Benth., Icon. Pl. 2: 87; pl. 1199. 1876. Tipo: Peru, San Martin, prope Tarapoto, Peruvia orientalis, VI/1855, R. Spruce 4400 (Lectótipo, aqui designado: K! [K000196301]; Isolectótipos W!, K!).

Distribuição: próximo aos Andes do Equador e do Peru. No protólogo de Anodiscus peruvianus, são citadas duas coletas (Ruiz \& Pavon s.n. e Spruce 4400). Segundo o site Tropicos (http://www.tropicos.org/Name/14000111), o lectótipo de A. peruvianus seria a duplicata de Spruce 4400 depositada no herbário $\mathrm{K}$, enquanto as dos herbários W e BM seriam isolectótipos; porém, não há indicação do autor dessa lectotipificação. Como Bentham, o autor da espécie, trabalhou no herbário K e o Icones Plantarum, onde ela foi publicada, trata de plantas selecionadas deste herbário, designa-se aqui o material Spruce 4400 depositado no herbário K (Barcode / id K000196301) como sendo o lectótipo de Anodiscus peruvianus e as duplicatas dos herbários W e $\mathrm{K}$, os isolectótipos; a duplicata do BM não foi encontrada.

Material selecionado: PERU. Loreto: Coronel Portillo, Lower Boqueron del Padre Abad. 480 m s.n.m., 25/VII/1964, P.C. Hutchison et al. 6040 (M, P).

3. Goyazia Taub., Bot. Jahrb. Syst. 21: 451. 1896. Tipo: G. rupicola Taub.

3.1. Goyazia rupicola Taub., Bot. Jahrb. Syst. 21: 451. 1896. Tipo: Brazil, Goiás, habitat in fissuris rupium in Serra
Dourada, s.d., E.H.G. Ule 3180 (Lectótipo, aqui designado: HBG! [HBG506569]).

Distribuição: Endêmica do Brasil, ocorre nos estados de Minas Gerais, Goiás e Tocantins.

O material citado na publicação original da espécie (Ule 3180) estaria depositado no herbário B, porém foi destruído durante a Segunda Guerra Mundial. No herbário HBG, existe uma duplicata deste material, que é designada aqui como lectótipo de Goyazia rupicola. Martins \& Silva (2000) citaram o material Ule 745 depositado no herbário R como um isótipo, uma vez que há coincidência na localidade e no mês da coleta, e Ule substituía os números de campo por números definitivos que eram usados nas publicações. Segundo as autoras o número 745 seria o número de campo do coletor. Entretanto, dificilmente se terá certeza sobre a correspondência entre esses números e, portanto, se as duas coleções (Ule 3180 e 745 ) se tratam de duplicatas de uma mesma coleta.

Material selecionado: BRASIL. Goiás: Serra Dourada, entre rochedos, I/1893. E.H.G. Ule 745 (R); Pirenópolis, Parque Estadual dos Pirineus, paredão rochoso em frente a casa de madeira abandonada, próximo à antena, $15^{\circ} 47^{\prime} 34,5^{\prime \prime}$, 4849'57,9"W, 1321 m s.n.m., 18/I/2005, A.O. Araujo et al. 503 (ESA).

4. Mandirola Decne., Rev. Hort. 20 (ser. 3, 2): 468. 1848. Tipo: M. multiflora (Gardner) Decne. [=Mandirola hirsuta (DC.) A.O.Araujo \& Chautems].

4.1. Mandirola hirsuta (DC.) A.O.Araujo \& Chautems, comb. nov. Achimenes hirsuta DC., Prodr. 7: 536. 1839, non Achimenes hirsuta Lindl. (1843). Trevirana hirsuta (DC.) D.Dietr., Syn. Pl. 3: 568. 1843. Gloxinia hirsuta (DC.) Wiehler, Selbyana 1(4): 386. 1976, nom. illeg. (homon. post.), non Gloxinia hirsuta Lindl. (1826). Gloxinia planalta Wiehler, Selbyana 6: 144. 1983. Tipo: Brazil, Mato Grosso, in Brasilia sylvaticis circa Cujaba, XII/1832, A.L.P.S. Manso s.n. (Holótipo G-DC.! [G00133267]).

Achimenes multiflora Gardner, Icon. Pl. 5: pl. 468. 1842. Trevirana multiflora (Gardner) Vis., Atti Congr. Sci. Ital. 1847: 558. 1847. Locheria multiflora (Gardner) Regel, Flora 31: 252. 1848. Mandirola multiflora (Gardner) Decne., Rev. Hort. 20 (ser. 3, 2): 468. 1848. Tipo: Brasil, Tocantins, Arraias, on dry banks on woods on the Serra de Santa Brida, and near Villa de Arrayas, III/1840, G. Gardner 3873 (Holótipo K!; Isótipos BM!, G!, E!, F!, $\mathrm{P}$ !, W!).

Mandirola multiflora var. hortorum Hanst., Linnaea 27: 716. 1856. Tipo: Bot. Mag. 69 (ser. 2, 16): pl. 3993.1843 (Lectótipo, aqui designado).

Distribuição: Concentrada na parte central do Bioma Cerrado, ocorrendo nos estados de São Paulo, Minas Gerais, Mato Grosso, Goiás, Tocantins, Bahia e Pará, além do Distrito Federal. 
Roalson et al. (2005a) restabeleceram Mandirola e propuseram a inclusão de três espécies no gênero: $M$. rupestris (Gardner) Roalson \& Boggan, M. ichthyostoma (Gardner) Seem. ex Hanst. e M. multiflora (Gardner) Decne. Eles consideraram M. multiflora o nome correto para Gloxinia planalta Wiehler e Achimenes hirsuta DC., porém Achimenes hirsuta é o nome mais antigo, justificando esta nova combinação em Mandirola.

Mandirola hirsuta possui ampla distribuição geográfica e morfologia variável, mas sua distinção das outras duas espécies do gênero ( $M$. rupestris e $M$. Ichthyostoma) não é clara. Araujo (2007) concluiu que, morfologicamente, esse complexo deve ser tratado como uma única espécie, porém o complexo não forma um grupo monofilético (dados parcialmente publicados em Araujo et al. 2010a) e a delimitação desses táxons continua incerta.

Hanstein (1856), ao comparar as duas variedades de $M$. multiflora, citou a variedade-tipo como sendo M. multiflora var. gardneriana e citou o trabalho de Hooker (1843) como referência para as duas variedades. Hanstein (1856) relatou, ainda na coluna "Autores e sinônimos" da tabela na página 716 , a variedade $a$ de Hooker como sendo a variedade-tipo (M. multiflora var. multiflora) e a variedade $\beta$ de Hooker como sendo a outra variedade (M. multiflora var. hortorum). Como nenhum material de herbário foi citado para a variedade $\beta$, designa-se aqui a prancha 3993 da obra de Hooker (1843) como lectótipo de M. multiflora var. hortorum (Fig. 1).

Material selecionado: BRASIL. Mato Grosso: Chapada dos Guimarães, trilha da Casa de Pedra para o Morro de São Jerônimo, próximo ao paredão, $15^{\circ} 26^{\prime} 16^{\prime \prime}$ ', 5550'14,9"W, 683 m s.n.m., 12/I/2004, A.O. Araujo et al. 458 (ESA).

5. Seemannia Regel, Gartenflora 4: 183. 1855, nom. cons., non Seemannia Hook. (1848), nom. inval. Fritschiantha Kuntze, Rev. Gen. Pl. 3(3): 241. 1898, nom. illeg. superfl. Tipo: S. ternifolia Regel [=Seemannia sylvatica (Kunth) Hanst.].

5.1. Seemannia gymnostoma (Griseb.) Toursark., Bol. Soc. Argent. Bot. 7(2): 135. 1958. Gloxinia gymnostoma Griseb., Pl. Lorentz.: 179 (Abh. kön. Ges. Wiss. Göttingen 19: 227). 1874. Achimenes gymnostoma (Griseb.) Fritsch, Nat. Pflanzenfam. 4(3b): 175.1894. Tipo: Argentina, Tucuman, Siambon, in sylvis subtropicis umbrosis, 06/III/1872, P.G. Lorentz 727 (Lectótipo, designado por Xifreda 1996: GOET!).

Achimenes heppielloides Fritsch ex Rusby, Bull. Torrey Bot. Club 23: 151. 1896. Tipo: Bolívia, s.d., M. Bang s.n. (Holótipo NY!).

Achimenes rusbyi Britton ex Rusby, Mem. Torrey Bot. Club 6: 95. 1896. Tipo: Bolívia, between Tipuani and Guanai, XII/1892, M. Bang 1721 (Lectótipo, aqui designado: NY! [NY312501]; Isolectótipos BM!, E!, G!, K!, M!, MO!, NY!, PH!, US!, W!, WU!, Z!).

Fiebrigia digitaliflora Fritsch, Bot. Jahrb. Syst. 50: 397. 1913. Tipo: Bolívia, Tarija, Pinos, 2300 m s.n.m., 10/III/1904,
K. Fiebrig 3124 (Lectótipo, aqui designado: B! [B10 0177784, Image ID 236216]; Isolectótipos BM!, F!, G!, $\mathrm{K}$ !, MO!, S!).

Distribuição: Face leste dos Andes, desde a Argentina, passando pela Bolívia, até o Peru.

Fritsch (1913) não indicou onde estaria depositado o material de Fiebrigia digitaliflora, mencionou que, quando o herbário não estivesse claramente citado, o material teria sido enviado para ele pelo herbario de B para identificação. Fritsch trabalhou no herbário GZU, mas nenhuma duplicata de Fiebrig 3124 foi encontrada neste herbário. No herbário $B$, existe uma exsicata desta coleção, mas sem nenhuma indicação de que teria sido examinada por Fritsch. Dessa forma, designa-se aqui este material, depositado no herbário B com o número Barcode B 100177784 e Image Id: 236216, como lectótipo de Fiebrigia digitaliflora.

$\mathrm{Na}$ descrição de Achimenes rusbyi, Rusby (1896) não indicou em qual herbário estaria depositado o material examinado. A coleção-tipo deste nome refere-se à coleção Bang 1721, que também recebeu a numeração Rusby 1102 em algumas duplicatas no herbário NY. O material Bang 1721 com o número de identificação 312501 ("barcode ID number") no herbário NY é aqui designado como lectótipo de A. rusbyi.

Material selecionado: ARGENTINA. Jujuy: Ledesma, Parque Nacional Calilegua, ruta 83, rumbo a Valle Grande, próximo al limite NW del parque, entre la Mesada y el limite NW del parque. 18/01/2002, V.S. Neffa et al. 757 (G, HUEFS, MBM).

5.2. Seemannia sylvatica (Kunth) Hanst., Linnaea 29: 540. 1859. Gesneria sylvatica Kunth, Nov. Gen. Sp. Pl. (quarto. ed.) 2: 393; fol. ed.: 316. 1818. Fritschiantha sylvatica (Kunth) Kuntze, Rev. Gen. Pl. 3(3): 241. 1898. Gloxinia sylvatica (Kunth) Wiehler, Selbyana 1: 33. 1975. Tipo: Peru, prov. Bracamonensis, inter urbem Jaen et fluvium Amazonum, alt. 230 hex. Floret Augusto, A.J.A. Bonpland s.n. (Holótipo P-Bonpl.!).

Gesneria oxyphylla DC., Prodr. 7: 531. 1839. Tipo: Peru, Peruvia oriental, subandina, 1834, E.F. Poeppig 1631 (Holótipo G-DC!; Isótipos BM!, F!, G!, GOET!, LE!, $\mathrm{P}$ !, W!).

Seemannia ternifolia Regel, Gartenflora 4: 183; pl. 122. 1855. Tipo: Gartenflora 4: pl. 122 . 1855 (Lectótipo, aqui designado).

Seemannia benaryi Regel, Gartenflora 23: 353; pl. 814. 1874. Fritschiantha benaryi (Regel) Kuntze, Rev. Gen. Pl. 3(3): 242. 1898. Tipo: America tropical, B. Roezl. s.n. (Holótipo LE!).

Seemannia uniflora Baill., Bull. Mens. Soc. Linn. Paris 1: 710. 1888. Fritschiantha uniflora (Baill.) Kuntze, Rev. Gen. Pl. 3(3): 242. 1898. Tipo: Bolívia, La Cordillera, s.d., H.A. Weddell 4031 (Lectótipo, aqui designado: P!). Achimenes albescens Rusby, Mem. Torrey Bot. Club 6: 94. 1896. Seemannia albescens (Rusby) Fritsch, Bot. Jahrb. 


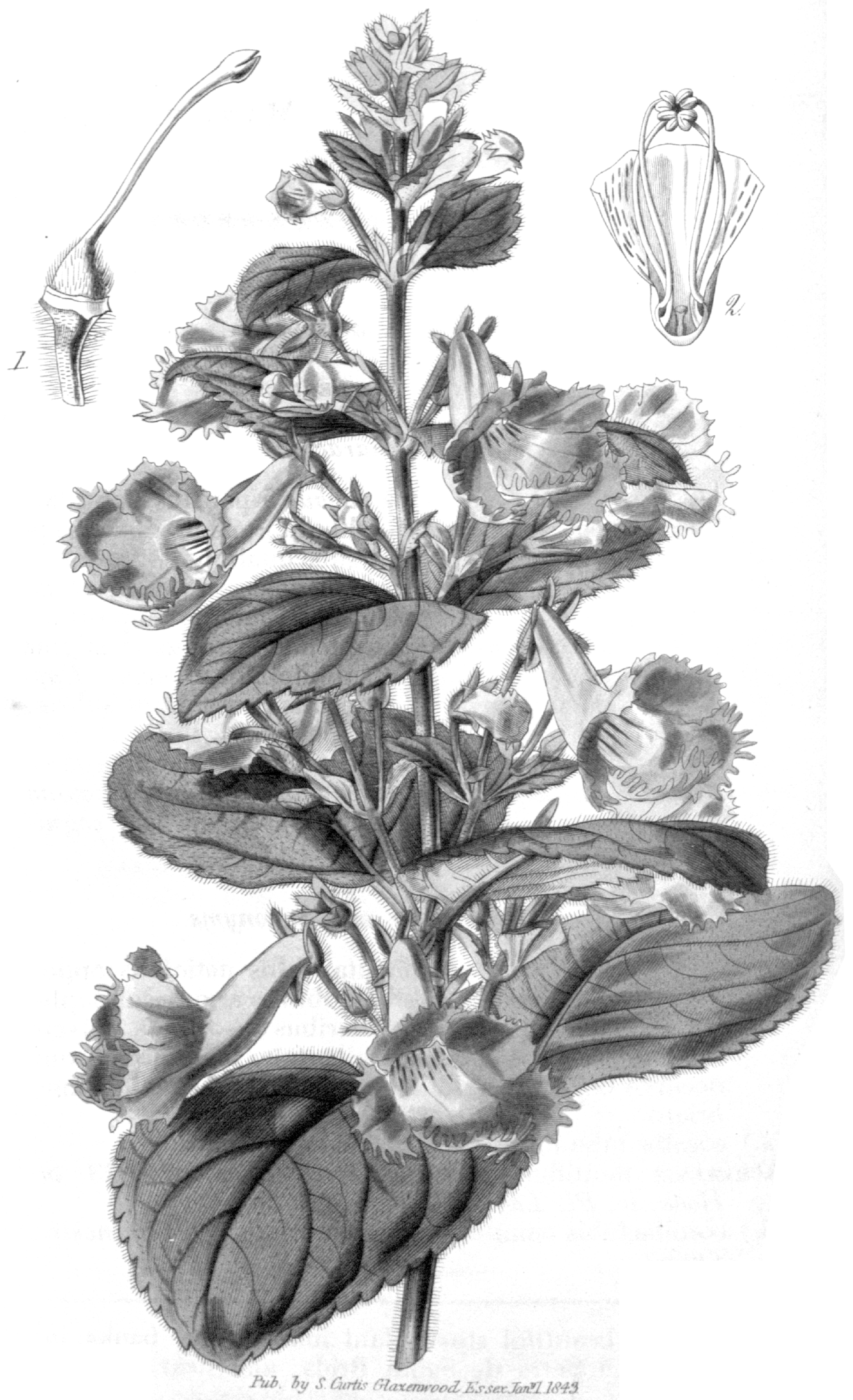

Figura 1. Ilustração de Mandirola multiflora var. hortorum Hanst., citada no protólogo (Hooker 1843: tab. 3993) e designado no presente trabalho como seu lectótipo. 
Syst. 50: 403. 1913. Tipo: Bolívia, Cochabamba, Espírito Santo, 1891, M. Bang 1211 (Lectótipo, aqui designado: NY [NY312496]!; Isolectótipos BM!, E!, K!, LE!, MO!, NY!, PH!, US!, W!, WIS!, Z!).

Seemannia cuneata Rusby, Mem. Torrey Bot. Club 6(1): 96. 1896. Fritschiantha cuneata (Rusby) Kuntze, Rev. Gen. Pl. 3(3): 242. 1898. Tipo: Bolívia, Cochabamba, s.d., $M$. Bang 1212 (Lectótipo, aqui designado: NY [NY313015]!; Isolectótipos BM!, BR!, E!, GH!, K!, M!, LE!, MICH!, NY!, US!, W!, WU!, Z!).

Fritschiantha sylvatica (Kunth) Kuntze var. coccinea Kuntze, Rev. Gen. Pl. 3(3): 241. 1898. Tipo: Bolívia, Yapacani, Sierra de Santa Cruz, 1800 m s.n.m., V/1892, O. Kuntze s.n. (Lectótipo, aqui designado: NY [NY622148]!; Isolectótipo NY!).

Fritschiantha sylvatica (Kunth) Kuntze var. aurea Kuntze, Rev. Gen. Pl. 3(3): 242. 1898. Tipo: Bolívia, Rio Juntas, 1400 m s.n.m., IV/1892, O. Kuntze s.n. (Lectótipo, aqui designado: NY [NY622145]!; Isolectótipo: NY!).

Seemannia regnelliana Fritsch, Bot. Jahrb. Syst. 29(Beibl. 65): 13. 1900. Tipo: Brasil, São Paulo, Cajuru, III/1857, A.F. Regnell III.951 a (Lectótipo, aqui designado: S [S05135]!; Isolectótipo S!).

Seemannia latifolia Fritsch, Meded. Rijks-Herb. 29: 52. 1916. Tipo: Bolívia, Quebrada de Cunucu, Ostkordillere, 600900 m s.n.m.; an quelligen, schattigen Stellen im Tälchen von los Monos, 1200 m s.n.m., III/1917, T. Herzog 1755 (Lectótipo, aqui designado: W!; Isolectótipos L!, S!, Z!). Distribuição: Cerrados, raramente em áreas de Floresta Ombrófila Mista das Regiões Sul, Sudeste e Centro-Oeste, alcançando as Florestas de Terras Baixas, de média e alta altitudes, da face Leste dos Andes, da Bolívia até o Equador, sendo encontrada raramente na face Oeste dos Andes peruanos.

Regel (1855) descreveu Seemannia ternifolia como uma erva perene com rizoma, sem ramificação, com tricomas curtos e rígidos por toda parte, de folhas lanceoladas, 3- ou 4-verticiladas, com margens inteiras, discolores, com nervuras salientes. Ele mencionou, ainda, que a planta estaria cultivada em estufa, e que seria proveniente das sementes enviadas por Warscewicz (nomeada como Gesneria quadrifolia), que a coletou no Peru. Rusby (1896) comentou que $S$. cuneata poderia ser diferenciada de $S$. ternifolia pelas folhas pecioladas cuneadas, pelo pedúnculo mais longo e mais rígido e pelas flores menores e mais estreitas. Tanto a descrição apresentada por Regel (1855) como a de Rusby (1896) são suficientemente claras para incluir S. ternifolia e S. cuneata na sinonímia de S. sylvatica. Regel (1874) citou que $S$. ternifolia seria parecida com $S$. benaryi, porém a primeira não contava com exsicatas para comparação. Essa referência reforça a ideia de que essa espécie (S. ternifolia) provavelmente não tenha sido descrita com base em material de herbário. Existe um material coletado por Regel e depositado no herbário $\mathrm{Z}$ - um dos possíveis locais onde os tipos de Regel estariam depositados - com o número 54.4, provavelmente indicando abril de 1854, ano anterior ao da publicação da espécie. Esta exsicata possui anotações com a letra do autor, está identificada como Seemannia ternifolia, e apresenta indicação de procedência do horto de Zurich (in sched.). O material é fiel à descrição e à ilustração presentes no protólogo, porém não foi citado na obra, não podendo ser considerado tipo deste nome. Dessa forma, a ilustração presente no protólogo é aqui designada como o lectótipo de Seemannia ternifolia (Fig. 2).

$\mathrm{Na}$ obra original de Seemannia uniflora, Baillon (1888) citou dois materiais: Weddell 4031 e d'Orbigny 1116. Em 1984, Skog anotou "lectotype of Seemannia uniflora Baill." no material de Weddell depositado no herbário P. No entanto, essa lectotipificação nunca foi publicada. O material d'Orbigny 1116 não foi encontrado nos herbários consultados, podendo ter ocorrido um erro no número indicado na obra original, já que foi encontrado o material d'Orbigny 1126 no herbário P. Dessa forma, o material Weddell 4031 (P) é aqui formalmente designado como lectótipo de Seemannia uniflora.

Kuntze (1898), ao descrever Fritschiantha sylvatica var. coccinea citou o material da seguinte forma: "Bolívia: Santa Rosa 2000 m, Sierra de Santa Cruz 1800 m, Yapacani 400 m." Cada uma dessas localidades da Bolívia corresponde a um material, ou ainda, alguns dos materiais misturam algumas dessas informações, principalmente referente a altitude. Dessa forma, tais materiais correspondem a síntipos desta variedade. No presente trabalho, designa-se o material Kuntze s.n (NY622148) como lectótipo de Fritschiantha sylvatica var. coccinea. Já para F. sylvatica var. aurea, Kuntze citou no protólogo: Bolívia 1800 $m$ Rio Juntas und San Antonio am Ostabhang der Anden mit $\alpha$ ( $\alpha$ significando a primeira variedade). Novamente, nenhum material foi encontrado com toda a informação do protólogo. Existem dois síntipos dessa variedade no herbário NY. Na ficha de coleta dos dois materiais, existe a informação: Bolívia, Rio Juntas, abril de 1892. Todavia, a altitude referida em um deles é $2000 \mathrm{~m}$ e no outro $1400 \mathrm{~m}$; porém, no protólogo é referido $1800 \mathrm{~m}$ alt. Esses dois materiais se enquadram perfeitamente na descrição apresentada por Kuntze para essa variedade. No presente trabalho, designa-se o material Kuntze s.n. (NY), com a seguinte localidade: Bolívia 1400 m, Rio Juntas, 13/21. abril 1892, com NY (ID 622145) como lectótipo de Fritschiantha sylvatica var. aurea.

Kuntze (1898) diferenciou a var. coccinea da var. aurea pela coloração da corola vermelho-alaranjada pintada de vermelho-escura (vs. corola amarelo-enxofre). Ele não citou a variedade típica, que teria a corola "coccinea", como descrito por Kunth na descrição da espécie, portanto muito semelhante à var. coccinea. Poderíamos supor que o autor estaria referindo-se a variedade-tipo da espécie quando descreveu F. sylvatica var. coccinea, porém o tipo citado no protólogo desta variedade não é o mesmo citado na descrição da espécie. Dessa forma, a variedade descrita por Kuntze seria sinônimo da variedade-tipo por não existir diferenças significativas para separá-las. Além disso, S. sylvatica apresenta uma grande variação na coloração da corola, que 


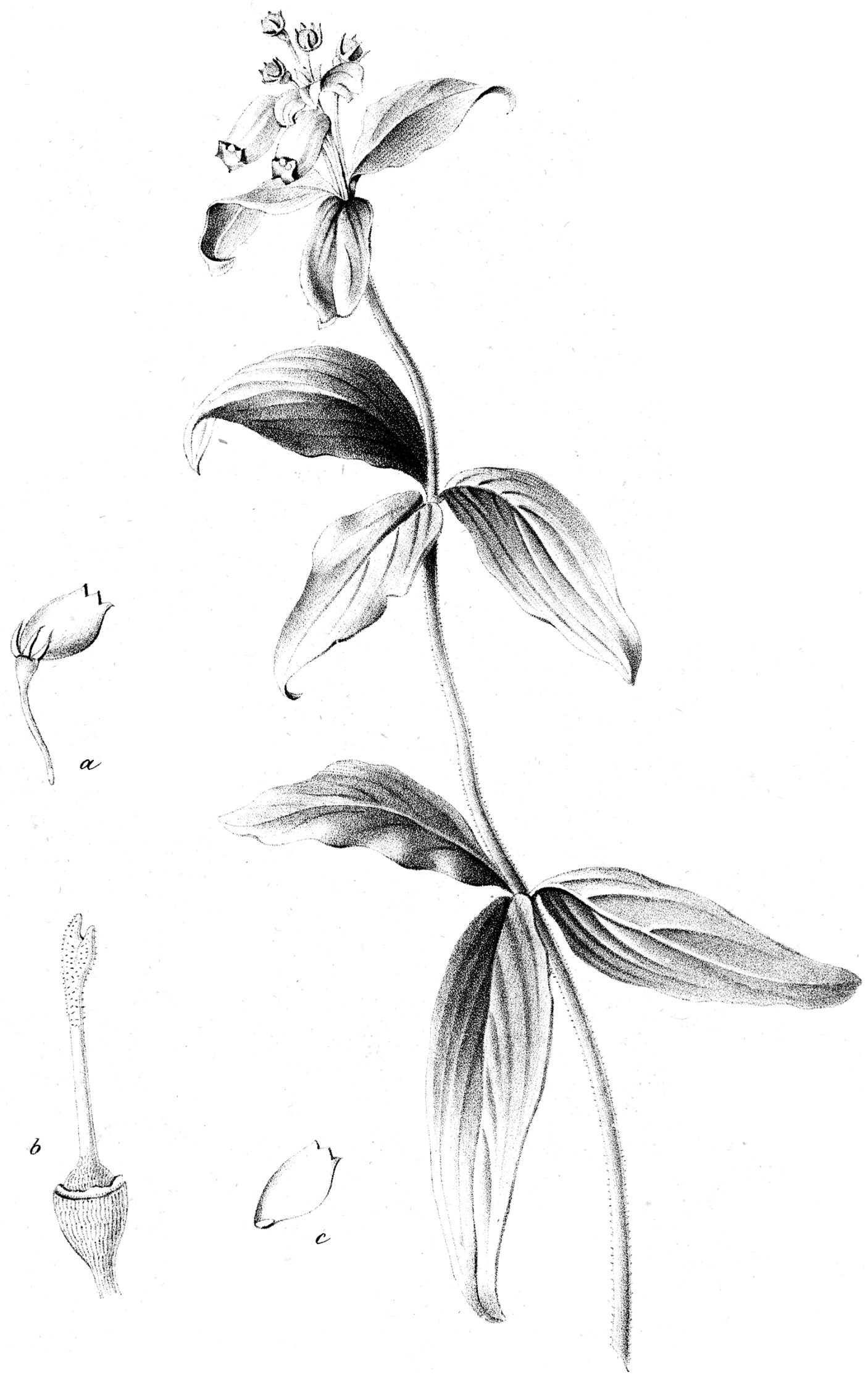

Figura 2. Ilustração de Seemannia ternifolia Regel, presente no protólogo (Regel 1855: tab. 122) e designada no presente trabalho como seu lectótipo. 
vai de vermelho-viva, passando por vermelho-alaranjada, chegando a amarela. Por esta razão, a outra variedade de Kuntze também não é aceita no presente trabalho.

As descrições originais de Achimenes albescens, Seemannia cuneata, S. regnelliana e S.latifolia não contam com a indicação dos holótipos. Dessa maneira, estamos designando aqui lectótipos para esses quatro nomes.

Material selecionado: BRASIL. Mato Grosso do Sul: Bonito, fazenda Baía das Garcas, cachoeira do rio Aquidaban, 550 m s.n.m. 12/XI/2002, G. Hatschbach et al. 74128 (MBM, SP).

\section{Incertae Sedis}

6.1. Seemannia major Baill., Bull. Mens. Soc. Linn. Paris 1: 710. 1888. Fritschiantha major (Baill.) Kuntze, Rev. Gen. Pl. 3(3): 242. 1898. Tipo: Bolívia, prov. de Larecaja et Caupolican (vallées entre Tripoani e Apolobamba), V/1847, H.A. Weddell 4549 (Holótipo P!).

Seemannia major foi considerada sinônimo de S. purpurascens por alguns autores (e.g., Skog \& Boggan 2011). No entanto, o tipo de $S$. major apresenta características intermediárias entre $S$. purpurascens e $S$. sylvatica, como está implícito nos comentários de Skog \& Boggan (2011), que sugerem uma origem híbrida para a espécie. $O$ único exemplar dessa espécie foi coletado no Departamento de La Paz (Bolívia), local onde a separação entre as espécies de Seemannia geralmente é difícil. Nesse material, existem anotações de Wiehler dizendo que se trata de um híbrido entre as duas espécies (S. purpurascens e S. sylvatica), sendo alguns ramos apontados como mais parecidos a $S$. sylvatica e outros como mais parecidos a $S$. purpurascens. Wiehler (1976) acabou considerando S. major sinônimo de S. sylvatica.

Na obra original de S. major, Baillon (1888, p. 710) descreveu:

"Muito próxima a S. sylvatica é S. major, erva boliviana coletada por Weddell (4549) nos vales entre Tripoani e Apolobamba. Altura 3 a $8 \mathrm{dm}$ e o caule simples, folhas lanceoladas, verticiladas por 3 ou 4 , mais raramente opostas na base do caule, $10 \times 4 \mathrm{~cm}$, membranácea, mole, esbranquiçada na face de baixo e lembrando aquelas de certas Gentianas pela aproximação de suas nervuras no verso na base do limbo. $A$ inflorescência terminal é curta, capituliforme (= o internó do ápice dos ramos é muito curto). A corola, tubular, com orifício oblíquo, atinge perto de $3 \mathrm{~cm}$ de compr.; toda pubescente. Os estames não apresentam 'espora'. $O$ estilete na extremidade do estigma fusiforme, oco, polposo no interior, como na espécie tipo; mas seu ápice é mais truncado e seus dois lobos são mais pronunciados. Weddell chamou a flor de velutina e escarlate". (tradução livre).
Apesar de morfologicamente mais parecido com S. sylvatica, o material Weddell 4549 apresenta caracteres intermediários que não se enquadram perfeitamente nessa espécie ou em S. purpurascens. Pelas características utilizadas para separar as duas espécies, $S$. major se aproxima mais de $S$. sylvatica, exceto pela coloração dos lobos da corola, que não é descrita na obra original de $S$. major. As folhas de S. major são lanceoladas ou oblanceoladas e a margem é inteira e geralmente revoluta; porém, às vezes, as folhas são elípticas, como pode ocorrer nas duas espécies, embora mais comumente em S. purpurascens. A aproximação das nervuras de S. major abaxialmente na base do limbo é bem parecida com o que ocorre frequentemente em $S$. sylvatica. Quanto às folhas, $S$. major mede $10 \times 4 \mathrm{~cm}$, sendo que, em S. sylvatica, elas nunca alcançam essa largura, enquanto, em $S$. purpurascens, elas podem chegar a $7,2 \mathrm{~cm}$. Já a corola de $S$. major aproxima-se de $3 \mathrm{~cm}$ compr., enquanto, em $S$. sylvatica, ela alcança até 2,5 $\mathrm{cm}$ compr. e, em S. purpurascens, ela pode chegar a $2,9 \mathrm{~cm}$ compr. Por outro lado, esse material não poderia ser tratado como uma terceira espécie, pois não apresenta características diagnósticas exclusivas. Tratá-la como um híbrido entre as duas espécies, como sugerido por Wiehler e Skog \& Boggan (2011), poderia ser uma solução. No entanto, os dados conhecidos ainda são insuficientes para tal afirmação. Por este motivo, este táxon é aqui tratado como incertae sedis.

6.2. Gloxinia suaveolens Decne., Rev. Hort. 20 (ser. 3, 2): 463. 1848. Tipo: Guianas, s.c. s.n.

Não foi encontrado nenhum material que correspondesse ao tipo de Gloxinia suaveolens. Wiehler (1976) e Skog \& Boggan (2011) consideraram G. suaveolens sinônimo de $G$. perennis. No entanto, a descrição original daquela espécie é bastante curta e não permite diferenciá-la com segurança de G. perennis: lacínios do cálice ovais, flores azul-pálidas e perfumadas. Ainda assim, existem características discordantes entre elas: em G. perennis, os lacínios do cálice são elípticos, às vezes espatuliformes, enquanto, em G. suaveolens, eles são ovais. Com base nas informações disponíveis, não foi possível reconhecer esta espécie, o que nos levou a considerá-la dentre aquelas com identidade incerta.

6.3. Seemannia longiflora Fritsch, Bot. Jahrb. Syst. 50: 404. 1913. Tipo: Peru, Sandia, 2300 m s.n.m., III/1902, A. Weberbauer 596 (B, destruído).

O holótipo de Seemannia longiflora foi destruído durante a Segunda Guerra Mundial. Fritsch (1913) apontou como diferença entre S. purpurascens e S. longiflora o comprimento das folhas, sendo que, na primeira, ele variaria de 6 a 12 $\mathrm{cm}$ e, na segunda, não ultrapassaria $5 \mathrm{~cm}$. De acordo com a descrição original de S. longiflora, tanto o comprimento quanto a largura das folhas são menores do que as encontradas em S. purpurascens por Araujo (2007): 4,1-16,3 x $1,5-7,2 \mathrm{~cm}$ (em S. purpurascens) e 2,5-5,0 x 1,2-1,5 cm (em S. longiflora). Além dessas diferenças, poderiam ser apontados também: o formato das folhas e dos lobos da corola, 
além da distribuição. Segundo a obra original, S. longiflora apresenta folhas elíptico-lanceoladas, lobos da corola curtamente arredondados e seria proveniente do Peru. Já as folhas de $S$. purpurascens são elíptico-ovais, os lobos da corola nunca são arredondados, mas sim triangulares, e a espécie ocorre no Brasil, Bolívia e Guiana. Wiehler (1976) e Skog \& Boggan (2011) sugeriram que $S$. longiflora seria sinônimo de S. purpurascens. Skog \& Boggan (2011) mencionaram que "[b]ased on type locality (Peru) this taxon is dubiously synonymized under S. purpurascens". No entanto, devido às diferenças apontadas acima eà falta de um material-tipo, $S$. longiflora é tratada como incertae sedis no presente trabalho.

\section{Agradecimentos}

Os autores agradecem aos curadores dos herbários consultados; ao apoio da Fundação de Amparo à Pesquisa do Estado de São Paulo, International Association for Plant Taxonomy e Elvin McDonald Research Endowment Fund of the Gesneriad Society, Inc; à biblioteca do Conservatoire et Jardin Botaniques de la Ville de Genève e a Manuel Faustino, pela disponibilização e pela digitalização das ilustrações das obras originais, respectivamente. Especial agradecimento ao Dr John Boggan e a dois outros revisores anônimos pelas preciosas contribuições.

\section{Referências}

André, E. 1873. Tydaea lindenii. Illustration Horticole 20 (sér. 3, 4): 183, pl. 147.

Araujo, A.O. 2007. Estudos taxonômicos em Gloxinia L'Hér. sensu lato (Gesneriaceae). Tese de Doutorado. Universidade de São Paulo, São Paulo.

Araujo, A.O.; Souza, V.C. \& Perret, M. 2010a. Chautemsia calcicola: A new genus and species of Gloxinieae (Gesneriaceae) from Minas Gerais, Brazil. Taxon 59: 203-208.

Araujo, A.O.; Souza, V.C. \& Perret, M. 2010b. Estudos taxonômicos em Gloxinia s.l. (Gesneriaceae) - I: uma nova espécie do Mato Grosso do Sul, Brasil. Rodriguésia 61(Supl.): S67-S72.

Baillon, H. 1888. Lorganisation florale des Seemannia. Bulletin Mensuel de la Société Linnéenne de Paris 1: 709-710.

Fritsch, K. 1913. Beitrag zur Kenntnis der Gesnerioideae. Botanische Jahrbücher für Systematik, Pflanzengeschichte und Pflanzengeographie 50(4): 397-398.
Hanstein, J. 1856. Die Gesneraceen des Koniglichen Herbariums und der Garten zu Berlin. Linnaea 27: 716-717.

Hanstein, J. 1864. Gesneriaceae. Pp. 342-428. In: Martius, C.F.P. (Ed.). Flora brasiliensis. Vol. 8. Leipzig, F. Fleischer.

Hooker, W.J. 1843. Achimenes multiflora. Botanical Magazine 69 [nov. ser.16 ]: 3993.

Hooker, W.J. 1846. Gloxinia pallidiflora. Botanical Magazine 72: tab. 4213.

Kuntze, O. 1898. Gesneraceae. Pp. 240-242. In: Revisio generum plantarum. Vol. 3(2). Leipzig, Arthur Felix.

L'Héritier de Brutelle, C.L. 1789. Gloxinia. Hortus Kewensis 2: 331.

Martins, V.L.C. \& Silva, I.M. 2000. Tipos das plantas vasculares do herbário do Museu Nacional (R) - XVII: Gesneriaceae. Boletim do Museu Nacional. Nova Série Botânica 108: 1-12.

Regel, E. 1849. Einige neue Gattungen der Gesnereen. Flora 32(12): $177-182$.

Regel, E. 1855. c) Seemannia ternifolia. In: I. Originalabhandlungen. 1) Abgebildete Pflanzen. Gartenflora 4(6): 183-184; pl. 122.

Regel, E. 1868. a) Tydaea lindeniana Rgl. In: I. Originalabhandlungen. 1) Abgebildete Pflanzen. Gartenflora 17(9): 257; pl. 589.

Regel, E. 1874. I. Originalabhandlungen. 1) Abgebildete Pflanzen. a) Seemannia benaryi. Gartenflora 23: 353; tab. 814.

Roalson, E.H.; Boggan, J.K. \& Skog, L.E. 2005a. Reorganization of tribal and generic boundaries in the Gloxinieae (Gesneriaceae: Gesnerioideae) and the description of a new tribe in the Gesnerioideae, Sphaerorrhizeae. Selbyana 25(2): 225-238.

Roalson, E.H.; Boggan, J.K.; Skog, L.E. \& Zimmer, E.A. 2005b. Untangling Gloxinieae (Gesneriaceae). I. Phylogenetic patterns and generic boundaries inferred from nuclear, chloroplast, and morphological cladistic datasets. Taxon 54: 389-410.

Rusby, H.H. 1896. A new Achimenes from Bolivia: Achimenes heppielloides Fritsch ex Rusby, n.sp. Bulletin of the Torrey Botanical Club 23: 151-152.

Skog, L.E. \& Boggan, J.K. 2011. World checklist of Gesneriaceae. Washington, DC, Smithsonian Institution. Disponível em: $<$ http://persoon. si.edu/gesneriaceae/checklist >. Acesso em: 4 de abril de 2012 .

Stearn, W.T. 1957. An introduction to the Species Plantarum and cognate botanical works of Carl Linnaeus. Pp. 1-176. In: Linnaeus, C. (Ed.). Species plantarum. A facsimile of the first edition 1753. Vol. 1. London, Ray Society.

Taubert, P. 1896. Gesneraceae. In: Beiträge zur Kenntnis der Flora des centralbrasilianischen Staates Goyaz. Botanische Jahrbücher für Systematik, Pflanzengeschichte und Pflanzengeographie 21: 451.

Wiehler, H. 1976. A report on the classification of Achimenes, Eucodonia, Gloxinia, Goyazia and Anetanthus (Gesneriaceae). Selbyana 1: 374-404.

Wiehler, H. 1983. A synopsis of the neotropical Gesneriaceae. Selbyana 6: 1-219.

Zimmer, E.A.; Roalson, E.H.; Skog, L.E.; Boggan, J.K. \& Idnurm, A. 2002. Phylogenetic relationships in the Gesnerioideae (Gesneriaceae) based on nrDNA ITS and cpDNA trnL-F and trnE-T spacer region sequences. American Journal of Botany 89: 296-311. 